\title{
A critical analysis of the models connecting molecular mass distribution and shear viscosity functions
}

\author{
L. Macskási* \\ Scientific Society of Mechanical Engineering, Fő utca 68, H-1027 Budapest, Hungary
}

Received 15 January 2009; accepted in revised form 9 April 2009

\begin{abstract}
Thermoplastics having various short and long-chain branches, characterized by the melt index measured at the processing temperature - according to their average molecular mass - can be processed using universal principles, independently of their chemical composition. The average molecular mass is the result of a molecular mass distribution, being the fingerprint of the chemical synthetic technology. The actual shape of the shear viscosity function aiming at the quantitative characterization of viscous flow, containing material-dependent parameters, depends on the ratio of high and low molecular mass fractions, the width of the molecular mass distribution function and on the number of short and long chain branches. This publication deals with the critical analysis of the mathematical methods of transforming these two curves of basic importance into each other.
\end{abstract}

Keywords: rheology, molecular mass, molecular mass distribution, shear viscosity function, direct and indirect transformation model

\section{Introduction}

\subsection{Establishing a research hypothesis}

Table 1 shows the stations of the technical-scientific quest during which a plastic product is made by cooling and shaping a melt produced by melting a polymer mass in the plastics processing industry. There are three separate, well-defined research areas: polymer structure research, testing the viscous flow properties of polymer melts, and testing the processing properties. First two are mainly scientific disciplines, the third is a practice-oriented activity. Our main interest was in establishing mutual relations between them, taking into account especially the works of Bersted [1-3], Malkin and Teishev [4-6], Tuminello and coworkers [7, 8] and Nobile et al. [9], who dealt first with determining the molecular mass distribution functions of thermoplastics from melt viscosity functions.

\section{Experimental}

\subsection{Materials}

The materials used in our experiments were

- polystyrenes of Edistir type (Dunasytr Plc, Hungary), samples denoted as PS 1-11

- PVC pellets of various K-values of Ongrolit type, (BorsodChem Plc, Hungary), samples denoted as PVC 1-6

- polypropylene homopolymers of Tipplen type (TVK Plc, Hungary), samples denoted as PP 1-6 The samples are listed in Table 2.

\subsection{Test methods}

\subsubsection{Determining the molecular mass distribution by GPC}

The molecular mass of polymers influences their general performance, such as tensile strength, elas- 
Table 1. Research hypothesis

\begin{tabular}{|c|c|c|c|c|c|c|c|c|}
\hline Polymers & $\mathbf{A B} / \mathbf{B A}$ & B & Polymer melts & BC & $\mathbf{C}$ & \multicolumn{3}{|c|}{ Processing properties } \\
\hline Molecular mass distribution & \multirow{3}{*}{ 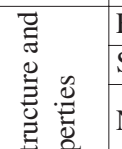 } & \multicolumn{2}{|c|}{ Flow curve } & \multirow[b]{2}{*}{ की० } & \multirow{3}{*}{\multicolumn{2}{|c|}{ Extrusion }} & \multicolumn{2}{|l|}{ Exit pressure } \\
\hline Molecular mass & & \multicolumn{2}{|c|}{ Shear viscosity function } & & & & \multicolumn{2}{|c|}{ Rheological swelling } \\
\hline Short chain branching & & \multicolumn{2}{|c|}{ Normal stress curve } & \multirow{2}{*}{ 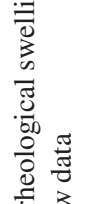 } & & & \multicolumn{2}{|c|}{$\begin{array}{l}\text { Elastic turbulence (melt } \\
\text { fracture) }\end{array}$} \\
\hline Long chain branching & \multirow{3}{*}{ 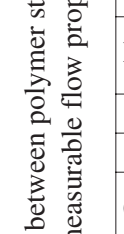 } & \multicolumn{2}{|c|}{ Normal viscosity function } & & \multicolumn{2}{|c|}{ Injection molding } & \multicolumn{2}{|c|}{$\begin{array}{l}\text { Internal pressure in the } \\
\text { mold }\end{array}$} \\
\hline Structural studies & & & eological tests & $\Xi \frac{3}{0}$ & \multicolumn{2}{|c|}{ Rheological swelling } & Yes & No \\
\hline $\begin{array}{l}\text { Gel permeation chromatog- } \\
\text { raphy }\end{array}$ & & \multicolumn{2}{|c|}{ Capillary viscometer } & 氙 & \multicolumn{2}{|c|}{ Processing method } & Extrusion & $\begin{array}{l}\text { Injection } \\
\text { molding }\end{array}$ \\
\hline X-ray diffraction & 异 & \multicolumn{2}{|c|}{ Rotation viscometer } & $\stackrel{\square}{ \pm}$ & \multicolumn{2}{|c|}{ Molecular mass } & $\begin{array}{l}\text { Medium and } \\
\text { high }\end{array}$ & $\begin{array}{l}\text { Low and } \\
\text { medium }\end{array}$ \\
\hline \multirow[t]{3}{*}{ Infrared spectroscopy } & 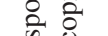 & \multicolumn{2}{|c|}{ Extrusiometer } & 60 & \multicolumn{2}{|c|}{ Molecular mass distribution } & Wide & Medium \\
\hline & : & \multicolumn{2}{|c|}{$\begin{array}{l}\text { Injection mold with built-in } \\
\text { sensors }\end{array}$} & 亶 & \multicolumn{2}{|c|}{ Temperature } & $\begin{array}{l}\text { Low and } \\
\text { medium }\end{array}$ & High \\
\hline & $\sum^{\frac{\sigma}{E}}=$ & \multicolumn{2}{|c|}{ Extensional rheometer } & ڤัँ & \multicolumn{2}{|c|}{ Production rate } & $\begin{array}{l}\text { Low and } \\
\text { medium }\end{array}$ & High \\
\hline
\end{tabular}

Table 2. Carreau parameters obtained by fitting the rheological test results of the polymers studied at $190^{\circ} \mathrm{C}$ by Equation (6) and the molecular mass distribution constants of Equation (1) obtained from the GPC data

\begin{tabular}{|c|c|c|c|c|c|c|c|c|c|c|c|}
\hline \multirow[t]{2}{*}{ Polymer } & \multicolumn{4}{|c|}{ Carreau parameters } & \multicolumn{4}{|c|}{ GPC test data } & \multicolumn{3}{|c|}{$\begin{array}{l}\text { Parameters of the molecular } \\
\text { mass distribution curve }\end{array}$} \\
\hline & $\eta_{0}[\mathrm{~Pa} \cdot \mathbf{s}]$ & $\lambda[\mathrm{s}]$ & $\mathbf{M}$ & $\mathbf{N}$ & $\overline{\mathbf{M}}_{\mathbf{m}}$ & $\overline{\mathbf{M}}_{\mathbf{n}}$ & $\overline{\mathbf{M}}_{\mathbf{m}} / \overline{\mathbf{M}}_{\mathbf{n}}$ & $\mathbf{M}_{\max }$ & $\alpha$ & $\sigma$ & $\mu$ \\
\hline \multicolumn{12}{|c|}{ EDISTIR polystyrene } \\
\hline PS-1 & 23451 & 0.930 & 0.771 & 0.773 & 162634 & 64072 & 2.538 & 132359 & 0.026503 & 0.6305 & 4.9559 \\
\hline PS-2 & 18131 & 0.774 & 0.760 & 0.749 & 157532 & 58431 & 2.696 & 124360 & 0.026422 & 0.6860 & 4.9116 \\
\hline PS-3 & 11762 & 0.580 & 0.983 & 0.553 & 160375 & 62151 & 2.580 & 150795 & 0.026831 & 0.6444 & 4.9172 \\
\hline PS-4 & 43449 & 1.433 & 0.891 & 0.713 & 176472 & 71525 & 2.467 & 138452 & 0.027770 & 0.5758 & 5.0266 \\
\hline PS-5 & 23955 & 0.951 & 0.825 & 0.711 & 189487 & 88165 & 2.149 & 160388 & 0.025332 & 0.5200 & 5.0492 \\
\hline PS-6 & 48157 & 2.208 & 0.777 & 0.779 & 158968 & 58993 & 2.695 & 128254 & 0.023147 & 0.7882 & 4.9204 \\
\hline PS-7 & 41720 & 1.369 & 0.763 & 0.816 & 131583 & 59117 & 2.226 & 102684 & 0.028333 & 0.6216 & 4.9437 \\
\hline PS-8 & 13197 & 0.551 & 0.873 & 0.677 & 150708 & 55342 & 2.723 & 124857 & 0.027397 & 0.6978 & 4.9101 \\
\hline PS-9 & 61515 & 2.673 & 0.865 & 0.701 & 187972 & 95438 & 2.873 & 151415 & 0.027990 & 0.7419 & 4.9958 \\
\hline PS-10 & 39817 & 1.564 & 0.816 & 0.768 & 150680 & 55797 & 2.701 & 112421 & 0.026919 & 0.5917 & 5.0165 \\
\hline PS-11 & 77149 & 4.110 & 0.714 & 0.842 & 150798 & 53184 & 2.835 & 124135 & 0.029322 & 0.5970 & 5.1160 \\
\hline \multicolumn{12}{|c|}{ ONGROLIT PVC } \\
\hline PVC-1 $(K=52)$ & 1432 & 0.861 & 0.426 & 0.362 & 80035 & 40049 & 1.998 & 68941 & 0.02585 & 0.5212 & 4.7364 \\
\hline PVC-2 $(K=58)$ & 7824 & 0.782 & 0.725 & 0.575 & 106986 & 52384 & 2.042 & 89182 & 0.02634 & 0.5292 & 4.8465 \\
\hline PVC-3 $(K=64)$ & 19759 & 0.481 & 0.988 & 0.586 & 145541 & 68544 & 2.123 & 119524 & 0.02768 & 0.5722 & 4.9804 \\
\hline PVC-4 $(K=67)$ & 32531 & 0.636 & 1.027 & 0.603 & 164508 & 80261 & 2.050 & 133922 & 0.02742 & 0.5160 & 5.0522 \\
\hline PVC-5 $(K=70)$ & 50453 & 0.677 & 1.445 & 0.483 & 188065 & 87418 & 2.151 & 160780 & 0.02919 & 0.5509 & 5.1185 \\
\hline PVC-6 $(K=77)$ & 167418 & 0.855 & 1.028 & 0.923 & 253923 & 114367 & 2.220 & 192797 & 0.02822 & 0.5481 & 5.2508 \\
\hline \multicolumn{12}{|c|}{ TIPPLEN polypropylene } \\
\hline PP-1 & 1491 & 0.076 & 0.845 & 0.679 & 212850 & 45274 & 4.701 & 110710 & 0.05481 & 0.8058 & 4.9795 \\
\hline PP-2 & 1470 & 0.066 & 0.901 & 0.672 & 219166 & 46200 & 4.744 & 118050 & 0.05740 & 0.7840 & 4.9762 \\
\hline PP-3 & 7479 & 0.448 & 0.724 & 0.791 & 269912 & 55980 & 4.822 & 158117 & 0.04550 & 0.7828 & 4.9858 \\
\hline PP-4 & 11009 & 0.706 & 0.912 & 0.618 & 336073 & 68097 & 4.935 & 188315 & 0.04519 & 0.8634 & 5.1434 \\
\hline PP-5 & 61349 & 2.826 & 1.288 & 0.468 & 480786 & 89048 & 5.399 & 319716 & 0.03139 & 0.8710 & 5.1959 \\
\hline PP-6 & 69067 & 1.821 & 1.067 & 0.570 & 617606 & 107993 & 5.719 & 526621 & 0.03371 & 0.9653 & 5.3282 \\
\hline
\end{tabular}

ticity modulus, while their molecular mass distribution plays a decisive role in their processing.

In order to determine the molecular mass distribution the polymer is usually dissolved and the so-called size-exclusion chromatogram (or gel permeation chromatogram, GPC) is determined. The are no known solvents for certain polymers (e.g. poly(tetrafluorethylene), poly(ether-ether-ketone)), while other can be studied only at high temperature (polyolefins). Solvent based techniques are usually time consuming not because of the GPC test islef, but because of the dissolution of the polymer sample. 
Solutions of about $5 \mathrm{mg} / \mathrm{ml}$ concentration were made from PS in tetrahydrofurane and from PVC in cyclohexanone at room temperature, while solutions of about $0.5-1 \mathrm{mg} / \mathrm{ml}$ concentration were made from PP in 1,2,4-trichlorobenzene at $160^{\circ} \mathrm{C}$, which were filtered on filters with $1 \mu \mathrm{m}$ pore diameter. Molecular mass distribution curves of PS and PVC samples were measured at room temperature using a Waters gel permeation chromatograph (GPC), while the molecular mass distribution of PP samples was determined by a Polymer Laboratories GPC 220 equipment at $160^{\circ} \mathrm{C}$. Polystyrene standards were used for calibration in each case.

\subsubsection{Determining the shear viscosity function}

Modern rheological test equipments (oscillating rheometers and capillary or rotation viscometers) make possible determining the frequency dependence of storage and loss moduli or the flow curves within 20 minutes and 1-2 hours respectively, covering 4 orders of magnitude frequency range or 6-8 orders of magnitude shear rate range respectively. They need neither solvent nor filtering. Therefore, rheological tests are ideally suited for quality control.

Mass and volume flows of the melts were measured using standard capillaries at $190^{\circ} \mathrm{C}$, using $1-21.6 \mathrm{~kg}$ load. Shear stress, shear rate and shear viscosity values were calculated from the test data.

\section{Quantitative description of the molecular mass distribution and critical analysis of determining their maximum shear viscosity}

\subsection{Mathematical description of the molecular mass distribution determined by GPC method}

Characteristic parameters of the distribution curve are determined by the GPC test software, namely the mass- and number average molecular masses and the molecular mass belonging to the maximum of the distribution function.

Mass average molecular mass of the polymers studied was in the range of $100000<\left(\bar{M}_{m}\right)<600000$ their polydispersity was moderate $\left(2<\bar{M}_{m} / \bar{M}_{n}<6\right)$. The functional form, i.e. the differential molecular mass distribution was described by the so-called log-normal distribution (Equation (1)): $y=\frac{a}{\sigma} \cdot \exp \left[-\frac{1}{2} \cdot \frac{\ln x-\mu}{\sigma^{2}}\right]$

which is strictly valid only for linear, monodisperse polymers, mainly for polyethylene [10], but can be used as a good approximation for commercial polymers (e.g. for polypropylene, polystyrene, and PVC samples [11]), provided that they do not contain too many branches and if the polydispersity is not too large. $a$ is a constant characterizing the maximum of the curve, $\sigma$ is the standard deviation of the distribution, $x$ is the molecular mass, $\mu$ is the molecular mass belonging to the maximum of the curve. Using the constants of Equation (1) the polydispersitiy index of the polymer is given by Equation (2):

$$
\frac{\bar{M}_{m}}{\bar{M}_{n}}=1+\left(\frac{\sigma}{\mu}\right)^{2}
$$

\subsection{Critical analysis of the determination of the maximum shear viscosity}

The shear viscosity function of polymer melts can be determined by a combined use of rotational and capillary rheometers in a broad shear rate range. Viscosity values close to the zero shear rate can be obtained by the former, while viscosities closer to the values encountered in practice can be obtained by the latter. Depending on the polymer and on the temperature it is possible to measure points at low shear rates $\left(\sim 0.1 \mathrm{~s}^{-1}\right)$ by capillary rheometers, but these are not typical.

The functional relation is usually described by functions containing 2, 3 or 4 parameters, resulting in gradually smaller fitting errors. Equations with 2 parameters cannot handle the Newtonian behavior, which can be described only by equations containing 3-4 parameters. One has to interpret the physical meaning of the parameters and the reality of the maximum viscosity value obtained.

The problem of determining the $\eta_{0}$ values lies in the fact that test data are determined in the so-called non-Newtonian range, and the usual, simpler extrapolation methods do not treat this range adequately.

When describing the shear viscosity as a function of the shear rate by Equation (3):

$\eta=\frac{1}{a_{1} \dot{\gamma}+b_{1}}$ 
and linearization $b_{1}=1 / \eta_{0}$ is yielded by the intercept.

Describing the shear viscosity by another function, namely Equation (4):

$\eta=\frac{\left(\eta_{0}\right)_{2}}{\dot{\gamma}^{a_{2}}}$

After linearization the shear viscosity belonging to $\dot{\gamma}=1 \mathrm{~s}^{-1}$ is the intercept, i.e. $\left(\ln \eta_{0}\right)_{2}$ and the shear viscosity increases monotonously and continuously with decreasing shear rates which is physically impossible.

When using a three-parameter Equation (5):

$$
\eta=\frac{\left(\eta_{0}\right)_{3}}{\left(\dot{\gamma}+b_{3}\right)^{a_{3}}}
$$

the horizontal section characteristic of the Newtonian range, i.e. the maximum shear viscosity appears in the logarithmic plot, but parameters $a_{3}$ and $b_{3}$ have no physical meaning.

The Carreau equation originally containing 3 parameters [12] has been modified to contain 4 parameters (see Equation (6)):

$$
\eta=\frac{\left(\eta_{0}\right)_{4}}{\left[1+\left(b_{4} \dot{\gamma}\right)^{c_{4}}\right]^{a_{4}}}
$$

which contains not only the maximum shear viscosity $\left(\eta_{0}\right)$ but also the relaxation time $\left(b_{4}=\lambda\right)$, as a material constant, a value $\left(c_{4}\right)$ characterizing the molecular mass distribution and a further parameter $\left(a_{4}\right)$ characterizing the non-Newtonian behavior can also be determined from it.

Figure 1 shows the curves fitted to shear viscosity data measured on PS-4 at $190^{\circ} \mathrm{C}$. We concluded, that at low shear rates Equation (4), while at high shear rates Equation (3) leads to erroneous conclu-

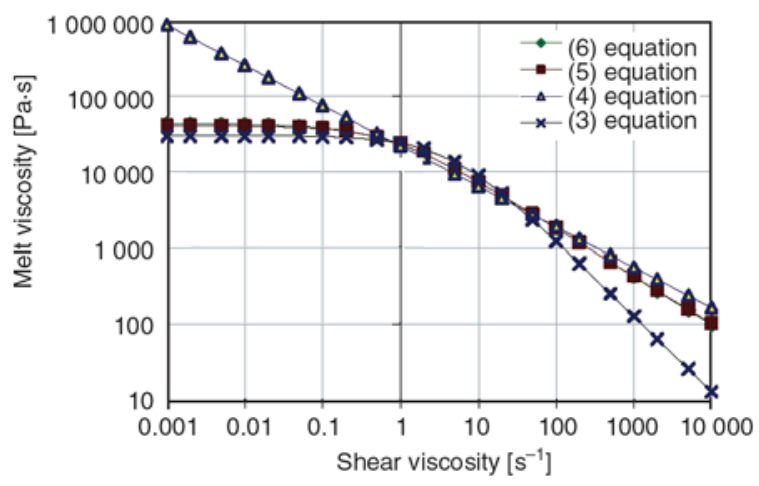

Figure 1. Determining the maximum shear viscosity of sample PS-4 by approximate equations at $190^{\circ} \mathrm{C}$ sions, reflected by the relatively large fitting errors (18.06 and 9.13\% respectively). Equations (5) and (6) can be well applied in the whole shear rate range, the fitting errors are only 2.38 and $2.15 \%$ respectively.

Carreau parameters of PS, PVC and PP samples determined form $190^{\circ} \mathrm{C}$ rheological tests are collected in Table 2 together with the parameters of the molecular mass distribution functions determined by GPC.

\subsection{Relation between the maximum shear viscosity and the mass average molecular mass}

\subsubsection{Literature data}

Calculation the maximum shear viscosity is of great theoretical importance, as it is directly related to the mass average molecular mass of the polymer, as shown by Equation (7) [13-15]:

$\eta_{0}=K_{1} \cdot \bar{M}_{m}^{\alpha}$

Parameters collected from the literature for HDPE, PS and PP are listed in Table 3. It has to be noted that $K_{1}$ parameters were obtained using zero shear viscosities using Poise units.

Figure 2 shows the relations between shear viscosities and mass average molecular masses for our PS,

Table 3. The constants of Equation (7) for various polymers at $190^{\circ} \mathrm{C}$

\begin{tabular}{|l|c|c|c|c|c|}
\hline & Polymer & $\mathbf{K}_{\mathbf{1}}$ & $\boldsymbol{\alpha}$ & $\mathbf{R}^{\mathbf{2}}$ & Reference \\
\hline \multirow{2}{*}{$\begin{array}{l}\text { Literature } \\
\text { data }\end{array}$} & HDPE & -12.700 & 3.5200 & & {$[16]$} \\
\cline { 2 - 6 } & $\mathrm{PS}$ & -12.800 & 3.4000 & & {$[17]$} \\
\cline { 2 - 6 } & $\mathrm{PP}$ & -11.900 & 3.6900 & & {$[18]$} \\
\hline \multirow{2}{*}{$\begin{array}{l}\text { Calculated } \\
\text { from test } \\
\text { data }\end{array}$} & $\mathrm{PS}$ & -32.545 & 7.0823 & 0.9964 & \\
\cline { 2 - 6 } & $\mathrm{PP}$ & -17.099 & 3.8211 & 0.9475 & \\
\cline { 2 - 6 } & $\mathrm{PVC}$ & -16.207 & 3.9698 & 0.9881 & \\
\hline
\end{tabular}

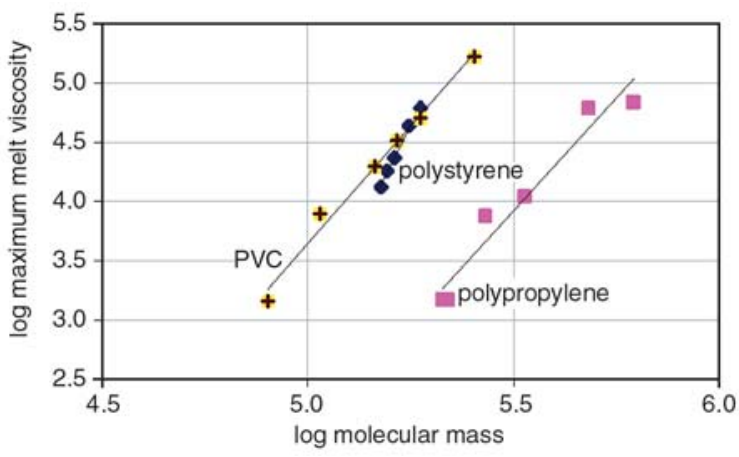

Figure 2. Maximum shear viscosities determined at $190^{\circ} \mathrm{C}$ plotted against the molecular mass 
PP and PVC samples. Zero shear viscosities are given here already in SI units.

The molecular mass range of the PS samples studied covers a very narrow range and, according to Equation (7) the slope is about double of the value obtained from the literature.

The exponent calculated for the polystyrene sample is too high, therefore the calculated molecular masses are far from the GPC results. The reason for this discrepancy is that the shear rated which can be realized in the capillary viscometer is not low enough to obtain a reliable zero shear viscosity by processing the data using the Carreau equation. There are two further methods which, however, require special instrument. Zero shear viscosity can be determined by oscillating rheometry. A third method uses the slope of the steady state of the deformation curve determined by creep experiments at low stresses.

\section{A model used for transforming molecular mass distribution curves into shear viscosity functions}

\subsection{Theory}

When establishing the relation between the polymer structure and macroscopically observed properties we started from the obvious fact that shear viscosity depends mainly of the chain length distribution, on the temperature and last, but not least on the shear rate $[19,20]$.

Bersted in his works [1-3] defined a critical molecular mass $M_{c}$, below which the molecular flow is regarded as Newtonian, while above this value the viscosity decreases with the shear rate. The critical molecular mass is essentially related to the Newtonian/non-Newtonian transition in the flow curve, which in its turn is related to the maximum relaxation time. Consequently the onset of the non-Newtonian flow is related to the longest relaxation time of the polymer sample.

Stated otherwise it is assumed that for each shear rate there exists a $M_{c}$ critical molecular mass. If $M<M_{c}$ the weight of contribution of molecules to the viscosity is proportional to $w_{i} M_{i}$, while if $M \geq M_{c}$ the contribution is proportional to their weight fraction, as if their molecular mass were $M_{c}$. It is further assumed that the threshold value is independent of the average molecular mass of the sample and of the molecular mass distribution of the whole sample.

\subsection{Calculation method}

In order to determine the critical molecular mass we start from the well known Equation (4) between maximum shear viscosity $\left(\eta_{0}\right)$ and mass average molecular mass $\bar{M}_{m}$ as described in linearized form by Equation (8):

$\lg \eta(\dot{\gamma})=K_{1}+\alpha \cdot \lg \bar{M}_{m}$

where Equation (9) defines the meaning of $\bar{M}_{m}$ :

$$
\bar{M}_{m}=\sum_{i=1}^{c-1} w_{i} M_{i}+M_{c} \sum_{i=c}^{\infty} w_{i}
$$

where $w_{i}$ is the mass fraction of the $i$-th component and $M_{i}$ is the molecular mass of the $i$-th component. The molecular mass distribution curve has been divided into $i=54$ discrete points (see Figure 3).

Rheological constants of the studied polymer types were determined form the shear viscosities measured at $190^{\circ} \mathrm{C}$ using the Carreau equation (see Equation (10)):

$\eta=\frac{\eta_{0}}{\left[1+(\lambda \cdot \dot{\gamma})^{M}\right]^{N}}$

wherein $\eta_{0}$ is the maximum shear viscosity in Pa.s units; $\lambda$ is the relaxation time [sec]; $M$ is a dimensionless constant characterizing the molecular mass distribution, while $N$ is another dimensionless constant characterizing the non-Newtonian behavior. At a given shear rate the 'apparent' molecular masses are calculated from Equation (8), from which the $M_{c}$ value is calculated using Equation (9).

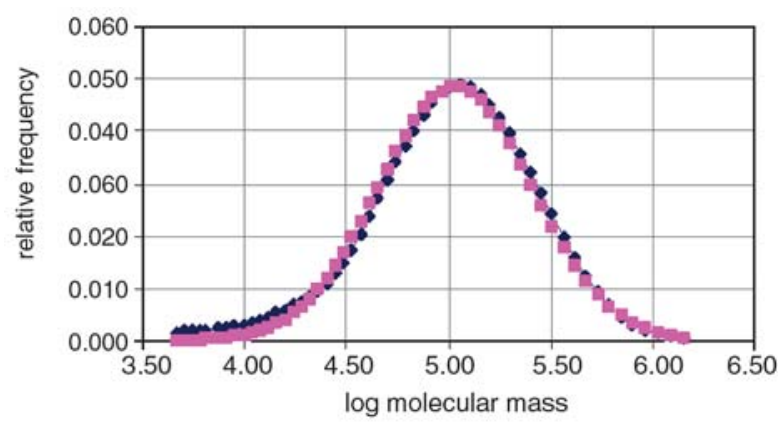

Figure 3. Molecular mass distribution of sample PS-4. 1 - GPC data, 2 - the curve fitted by Equation (1) 
Table 4. Calculation of critical molecular mass of sample PS-4

\begin{tabular}{|c|c|c|c|c|c|c|c|c|c|c|c|}
\hline \multirow{2}{*}{$\begin{array}{c}\text { Shear } \\
\text { rate }\left[s^{-1}\right]\end{array}$} & \multirow{2}{*}{$\begin{array}{c}\text { Viscosity } \\
{[\text { Pa } \cdot s]}\end{array}$} & \multirow{2}{*}{$\begin{array}{c}\text { Molecular } \\
\text { mass }\end{array}$} & \multicolumn{9}{|c|}{$\mathbf{M}_{\mathrm{c}}$ Critical molecular mass } \\
\hline & & & $\mathbf{i}=\mathbf{3 0}$ & $\mathbf{i}=\mathbf{3 3}$ & $\mathbf{i}=34$ & $i=35$ & $i=36$ & $\mathrm{i}=37$ & $\mathbf{i}=38$ & $\mathbf{i}=39$ & $i=40$ \\
\hline 1 & 18334 & 205458 & 307909 & 372962 & 403199 & 439192 & 482179 & 534168 & 597966 & 677532 & 777749 \\
\hline 10 & 5921 & 147352 & 212793 & 249068 & 264817 & 282740 & 303060 & 326200 & 352689 & 383174 & 418180 \\
\hline 20 & 4074 & 132004 & 187670 & 216343 & 228266 & 241415 & 255748 & 271268 & 287902 & 305423 & 323205 \\
\hline 100 & 1690 & 101906 & 138401 & 152168 & 156587 & 160376 & 162967 & 163545 & 160853 & 152951 & 136955 \\
\hline 1000 & 477 & 70237 & 86561 & 84643 & 81166 & 75106 & 65343 & 50198 & 27171 & -7480 & -59018 \\
\hline 2000 & 326 & 62788 & 74367 & 68760 & 63426 & 55049 & 42381 & 23537 & -4273 & -45216 & -105113 \\
\hline 5000 & 197 & 54138 & 60209 & 50318 & 42827 & 31761 & 15718 & -7420 & -40783 & -89033 & -158636 \\
\hline 10000 & 134 & 48396 & 50809 & 38074 & 29152 & 16300 & -1983 & -27972 & -65023 & -118122 & -194170 \\
\hline
\end{tabular}

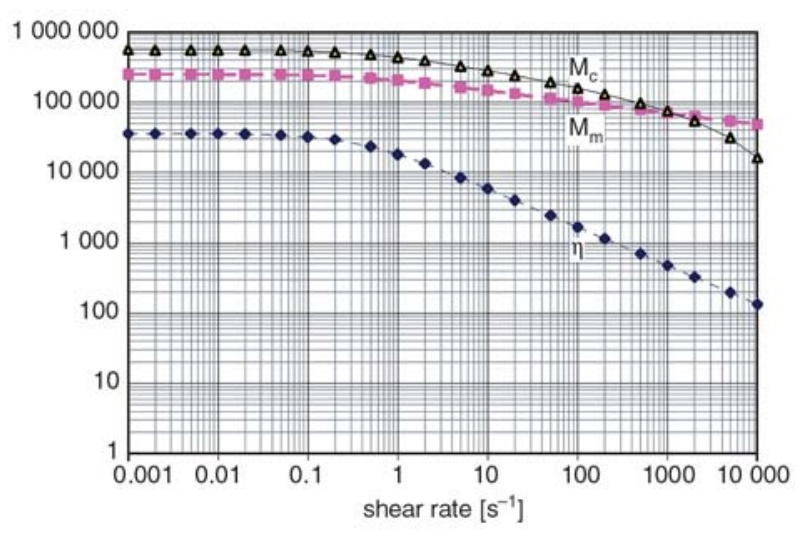

Figure 4. Shear viscosity ( $\eta$, in Pa·s units), molecular mass $\left(M_{m}\right)$ and critical molecular mass $\left(M_{c}\right)$ of sample PS-4 as a function the shear rate

When determining the $M_{c}$ values it was observed that after a certain $i$ value $M_{c}$ becomes negative, which is physically meaningless. Therefore an $i=c$ value was adopted, where a positive $M_{c}$ was obtained even at the highest shear rate, i.e. $i=35$ (Table 4).

When plotting the logarithm of the $M_{c}$ values against the logarithm of the shear rate a monotonically decreasing curve was obtained. Using the slope of the latter the following Equation (11) was used to calculate the $M_{c}$ values:

$M_{c}=M_{c, 0} \cdot \dot{\gamma}^{-1 / 4}$

where $M_{c, 0}=320000$ is used for PS [1].

A serious drawback of Equation (11) is that it approximates the measured shear viscosity function only in the non-Newtonian rage, but it cannot be used to calculate the Newtonian range. We tried to eliminate this deficiency first by a relation of $M_{c}=$ $a /(\dot{\gamma}+b)$ but a good result was obtained only by using a relation of $M_{c}=A /\left[1+(B \cdot \dot{\gamma})^{C}\right]^{D}$ (see Figure 4).

\subsection{Results and discussion}

The molecular mass distribution is transformed into the shear viscosity function in the following way:

(1) knowing the average molecular mass and the molecular mass distribution curve of the polymer one calculates $A=M_{c}$ at zero shear rate using Equation (12):

$$
A=M_{c(\dot{\gamma}=0)}=\frac{\bar{M}_{m}-\sum_{i=1}^{c-1} w_{i} M_{i}}{\sum_{i=c}^{\infty} w_{i}}
$$

for an $i=c$ value as close as possible to the average molecular mass.

(2) then the points of the shear viscosity function are calculated using Equation (13):

$\eta=\exp \left\{K_{1}+\alpha \cdot \lg \left[\sum_{i=1}^{c-1} w_{i} M_{i}+\frac{A}{\left(B \cdot \dot{\gamma}^{C}+1\right)^{D}} \cdot \sum_{i=c}^{\infty} w_{i}\right]\right\}$

Table 5 summarizes the data obtained from the GPC curves of the PS, PVC and PP samples, the constants of the measured and calculated shear viscosity functions and their deviations. Relative differences, $\sum_{i} 100 \cdot\left[\left(\left|\eta_{\text {measured }}-\eta_{\text {calculated }}\right|\right) / \eta_{\text {measured }}\right]$ are mostly within $10 \%$.

\section{Model used to determine molecular mass distribution of thermoplastic polymers from dimensionless rheological data}

\subsection{Theory}

Having discussed the direct problem (i.e. the determination of the flow curve from a known molecular mass distribution) in Chapter 4 the following question is asked: is it possible to calculate the molecu- 
Table 5. Parameters of the measured and calculated shear viscosity functions of the polymers tested and their relative differences

\begin{tabular}{|c|c|c|c|c|c|c|c|c|c|c|c|c|}
\hline \multirow[b]{2}{*}{ Polymer } & \multicolumn{3}{|c|}{ GPC curve } & \multicolumn{4}{|c|}{ Shear viscosity function (measured) } & \multicolumn{4}{|c|}{ Shear viscosity function (calculated) } & \multirow{2}{*}{$\begin{array}{l}\text { Rel. } \\
\text { diff. } \\
{[\%]}\end{array}$} \\
\hline & $\mathbf{i}=\mathbf{c}$ & $\sum_{i=c}^{\infty} w_{i}$ & $\sum_{i=1}^{c-1} w_{i} M_{i}$ & $\begin{array}{c}\eta \\
{[\mathrm{Pa} \cdot \mathrm{s}]}\end{array}$ & $\begin{array}{c}\lambda \\
{[\mathrm{s}]}\end{array}$ & $\begin{array}{l}\mathbf{M} \\
{[-]}\end{array}$ & $\begin{array}{l}\mathbf{N} \\
{[-]}\end{array}$ & $\mathbf{A}$ & B & $\mathbf{C}$ & D & \\
\hline \multicolumn{13}{|c|}{ EDISTIR polystyrene } \\
\hline PS-1 & 36 & 0.2991 & 47019 & 23451 & 0.930 & 0.771 & 0.773 & 517979 & 0.2059 & 0.5658 & 0.8393 & 8.093 \\
\hline PS-2 & 34 & 0.3896 & 40387 & 18131 & 0.774 & 0.760 & 0.749 & 429558 & 0.2289 & 0.4765 & 0.9533 & 13.247 \\
\hline PS-3 & 34 & 0.3661 & 44471 & 11762 & 0.580 & 0.983 & 0.553 & 377485 & 0.1500 & 0.5198 & 1.0569 & 6.852 \\
\hline PS-4 & 35 & 0.3714 & 42342 & 43449 & 1.433 & 0.891 & 0.713 & 590576 & 0.3540 & 0.4137 & 1.0686 & 8.244 \\
\hline PS-5 & 29 & 0.4685 & 40725 & 23955 & 0.951 & 0.825 & 0.711 & 397664 & 0.2447 & 0.4614 & 1.0153 & 6.392 \\
\hline PS-6 & 34 & 0.4877 & 32162 & 48157 & 2.208 & 0.777 & 0.779 & 512694 & 0.4319 & 0.4058 & 0.9238 & 6.029 \\
\hline PS-7 & 36 & 0.2954 & 49504 & 41720 & 1.369 & 0.763 & 0.816 & 699714 & 0.2205 & 0.4054 & 1.4811 & 8.838 \\
\hline PS-8 & 34 & 0.3842 & 38520 & 13197 & 0.551 & 0.873 & 0.677 & 390846 & 0.1582 & 0.4974 & 1.0616 & 6.545 \\
\hline PS-9 & 34 & 0.4143 & 43666 & 61515 & 2.673 & 0.865 & 0.701 & 647189 & 0.3035 & 0.3638 & 1.4798 & 8.619 \\
\hline PS-10 & 36 & 0.3242 & 47493 & 39817 & 1.564 & 0.816 & 0.768 & 638157 & 0.2900 & 0.4514 & 1.1377 & 10.236 \\
\hline PS-11 & 26 & 0.3502 & 54752 & 77149 & 4.110 & 0.714 & 0.842 & 635773 & 0.2645 & 0.4005 & 1.4376 & 10.369 \\
\hline average & & & & & & & & 530694 & 0.2593 & 0.4510 & 1.1323 & \\
\hline \multicolumn{13}{|c|}{ ONGROLIT PVC } \\
\hline PVC-1 & 38 & 0.1093 & 52452 & 1432 & 0.8613 & 0.4262 & 0.3618 & 210222 & 0.1614 & 0.3754 & 1.3456 & 1.905 \\
\hline PVC-2 & 32 & 0.3014 & 39808 & 7824 & 0.7817 & 0.7252 & 0.5751 & 253643 & 0.2355 & 0.5238 & 0.6539 & 4.033 \\
\hline PVC-3 & 29 & 0.4116 & 37204 & 19759 & 0.4814 & 0.9878 & 0.5859 & 265778 & 0.1747 & 0.6005 & 0.6584 & 5.677 \\
\hline PVC-4 & 28 & 0.4598 & 36431 & 32531 & 0.6361 & 1.0273 & 0.6028 & 285609 & 0.6165 & 0.5507 & 0.7320 & 6.480 \\
\hline PVC-5 & 27 & 0.4941 & 35451 & 50453 & 0.6770 & 1.4448 & 0.4833 & 303241 & 0.1922 & 0.8042 & 0.4917 & 9.247 \\
\hline PVC-6 & 24 & 0.6382 & 25541 & 167418 & 0.8545 & 1.0281 & 0.9231 & 360242 & 0.2748 & 0.5590 & 0.8721 & 11.649 \\
\hline average & & & & & & & & 279789 & 0.2759 & 0.5689 & 0.7923 & \\
\hline \multicolumn{13}{|c|}{ TIPPLEN polypropylene } \\
\hline PP-1 & 32 & 0.2207 & 71954 & 1491 & 0.0762 & 0.8454 & 0.6795 & 591533 & 0.04397 & 0.6027 & 1.0503 & 4.206 \\
\hline PP-2 & 32 & 0.2271 & 71143 & 1470 & 0.0655 & 0.9006 & 0.6720 & 574811 & 0.03294 & 0.6099 & 1.2131 & 4.708 \\
\hline PP-3 & 34 & 0.2010 & 112249 & 7479 & 0.4478 & 0.7236 & 0.7911 & 980237 & 0.03764 & 0.5296 & 1.3421 & 2.860 \\
\hline PP-4 & 31 & 0.3464 & 79132 & 11009 & 0.7061 & 0.9115 & 0.6180 & 768597 & 0.19658 & 0.4688 & 0.9415 & 6.047 \\
\hline PP-5 & 31 & 0.4442 & 87236 & 61349 & 2.8264 & 1.2884 & 0.4681 & 1066568 & 0.30630 & 0.3872 & 1.1782 & 11.270 \\
\hline PP-6 & 30 & 0.4604 & 112572 & 69067 & 1.8207 & 1.0668 & 0.5705 & 988648 & 0.23650 & 0.4343 & 1.2560 & 9.704 \\
\hline average & & & & & & & & 828399 & 0.14230 & 0.5054 & 1.1635 & \\
\hline
\end{tabular}

lar mass distribution from the shear viscosity function? This so-called 'inverse problem' is regarded as 'ill-defined' by Malkin and Teishev [4-6], Tuminello and coworkers [7, 8] and Nobile et al. [9]. Although molecular mass distribution curves calculated from low amplitude dynamic oscillation tests have been published in the literature, the algorithm has not been published [21,22].

Typical industrial polymers are polydisperse, i.e. they can be regarded as mixtures of monodisperse fractions. Flow curves/shear viscosity functions of monodisperse polymer melts can be described by Equation (14):

$\eta(\dot{\gamma})=\left\{\begin{array}{lllll}\eta_{0} & \text { if } & \tau \leq \tau_{\mathrm{s}} & \text { or } & \dot{\gamma} \leq \dot{\gamma}_{s} \\ \tau_{s} & \text { if } & \tau>\tau_{\mathrm{s}} & \text { or } & \dot{\gamma}>\dot{\gamma}_{s}\end{array}\right.$

where $\eta_{0}$ is the maximum shear viscosity, $\tau_{s}$ the critical shear stress and $\dot{\gamma}_{s}=\tau_{s} / \eta_{0}$ is the critical shear rate depending on the molecular mass of the high molecular mass fractions.

The molecular mass dependence of $\eta_{0}$ is given by Equation (7) wherein $K$ and $\alpha$ are constants ( $\alpha=$ 3.4-3.5). The critical shear rate can be calculated by Equation (15):

$\dot{\gamma}_{s}=\frac{\tau_{s}}{\eta_{0}(M)}=\frac{\tau_{s}}{K \bar{M}^{\alpha}}$

Equation (15) is strictly valid only for monodisperse polymers, for polydisperse samples mass average molecular mass $\bar{M}_{m}$ should be used. For a binary mixture of homodisperse polymers with $M_{1}$ and $M_{2}\left(M_{1}>M_{2}\right)$ Friedman and Porter [23] introduced the following Equation (16):

$\eta_{0, \text { combination }}=K \bar{M}_{m}^{\alpha}=\left(w_{1} \eta_{0,1}^{1 / \alpha}+w_{2} \eta_{0,1}^{1 / \alpha}\right)^{\alpha}$

where $\eta_{0,1}$ and $\eta_{0,2}$ are Newtonian viscosities and $w_{1}, w_{2}$ are the mass fractions of the components. 
The flow curve/viscosity function of the binary mixture can be expressed by Equation (17):

$$
\eta(\gamma)= \begin{cases}K\left(w_{1} M_{1}+w_{2} M_{2}\right)^{\alpha} & \text { if } \quad \dot{\gamma} \leq \frac{\tau_{s}}{K M_{1}^{\alpha}} \\ {\left[w_{1}\left(\frac{\tau_{s}}{\dot{\gamma}}\right)^{1 / \alpha}+w_{2} K^{1 / \alpha} M_{2}\right]^{\alpha}} & \text { if } \quad \frac{\tau_{s}}{K M_{1}^{\alpha}} \leq \dot{\gamma} \leq \frac{\tau_{s}}{K M_{2}^{\alpha}} \\ \frac{\tau_{s}}{\dot{\gamma}} & \text { if } \quad \dot{\gamma} \geq \frac{\tau_{s}}{K M_{2}^{\alpha}}\end{cases}
$$

This approximation has been generalized by Nobile et al. [9] for a continuous molecular mass distribution as shown by Equation (18):

$$
\begin{aligned}
& \eta(\dot{\gamma})= \\
& {\left[\int_{0}^{M(\dot{\gamma})}\left(K M^{a}\right)^{1 / \alpha} f(M) \mathrm{d} M+\left(\frac{\tau_{s}}{\dot{\gamma}}\right)^{1 / \alpha} \int_{M(\dot{\gamma})}^{\infty} f(M) \mathrm{d} M\right]^{\alpha}}
\end{aligned}
$$

where $f(M)$ is the molecular mass distribution function.

The first member of Equation (18) corresponds to the second member of Equation (17b) while its second member corresponds to the first member of Equation (17b). The first member of Equation (18) represents the Newtonian flow, while its second member is related to those high molecular mass fractions, which take part in the non-Newtonian flow.

In order to solve the inverse problem three dimensionless variables are needed, namely

- dimensionless shear viscosity according to Equation (19):

$Y=\left[\frac{\eta(\dot{\gamma})}{\eta_{0}}\right]^{1 / \alpha}$

- dimensionless shear rate according to Equation (20):

$$
X=\left(\frac{\dot{\gamma}}{\dot{\gamma}_{s}}\right)^{1 / \alpha}
$$

- and dimensionless molecular mass according to Equation (21):

$$
m=\frac{1}{X}=\frac{M}{\bar{M}_{m}}
$$

where the molar masses belonging to certain shear rates should be used instead of $M$, as shown by Equation (22):

$$
M=\frac{10^{\frac{1}{\alpha}\left(\lg \eta_{0}+K\right)}}{\left(\frac{\lambda \dot{\gamma}}{k}\right)^{1 / \alpha}}
$$

where $\eta_{0}$ is the maximum shear viscosity, $K$ is a constant characterizing the polymer, $\lambda$ is the relaxation time, $\dot{\gamma}$ is the actual shear rate and $k=1$.

Without presenting the derivation the solution of the inverse problem is given by Equation (23):

$$
f\left(\frac{1}{X}\right)_{\frac{1}{X}=m}=-\left(X^{4} \frac{\mathrm{d}^{2} Y}{\mathrm{~d} X^{2}}+2 X^{3} \frac{\mathrm{d} Y}{\mathrm{~d} X}\right)
$$

\subsection{Calculation of the molecular mass distribution}

Equation (23) can be made appropriate for calculations if the Carreau equation modified by us is inserted into Equation (24):

$$
Y=\frac{1}{\left[1+\left(k X^{\alpha}\right)^{M}\right]^{N / \alpha}}
$$

then it is derived twice with respect to $X$ as shown in Equation (25):

$$
\begin{aligned}
\frac{\mathrm{d}^{2} Y}{\mathrm{~d} X^{3}} & =M N k^{M} X^{\alpha M-2} \cdot \\
& \frac{M(N+\alpha) k^{M} X^{\alpha M}-(\alpha M-1)\left(1+k^{M} X^{\alpha M}\right)}{\left(1+k^{M} X^{\alpha M}\right)^{(N / \alpha+2)}}
\end{aligned}
$$

Using Equation (25) the frequency of each molecular mass in any polymer sample can be determined as a function of the shear rates used in the rheologi- 
cal test if the Carreau constants $(\lambda, M, N)$, and the constants of the given polymer $(k$ and $\alpha)$ are known.

\subsection{Results and discussion. Comparison of calculated and measured molecular mass distributions}

GPC curves are usually taken at points equally distributed along the log (molecular mass) axis, therefore it would be useful to distribute uniformly the test points along a logarithmic scale for melt viscosity tests too. The latter is hampered by the fact that even with up-to-date capillary rheometers one can take 6-8 (10) test point pairs (depending on the conditions). Intermediate points in the measured shear rate interval can be estimated by interpolation using the fitted shear viscosity function. A major problem is that in the case of $\dot{\gamma}<0.1 \mathrm{~s}^{-1}$ test are impossible, or even if one can measure 1-2 points for low viscosity melts, the scatter of parallel points will be unacceptably high. The same is true for tests at $\dot{\gamma}>100 \mathrm{~s}^{-1}$ where short test times do not allow the establishment of stationary flow. Additionally one cannot maintain the constant test temperature because of fictional heating. Thus the shear viscosity function must be extrapolated above and below the tested interval in order to estimate the high and low molecular mass branches of the molecular mass distribution function.

We could not describe the breadth of the distribution using the algorithm developed for the indirect problem. For a long time the Carreau parameters were blamed for this failure, but without success. Later we concentrated on the effect of varying $k$ and especially $\alpha$ which brought us closer to the solution of the problem.

The lack of success was due to the improper exponent value in equation $\eta_{0}=K \bar{M}_{m}^{\alpha}$. We stuck too much to the $\alpha=3.4$ regarded as universal in the literature which made impossible the approximately correct prediction of the molecular mass distribution. If a smaller $\alpha$ value is chosen, the lower (high molecular mass) and higher (low molecular mass) portions of the distribution function can be approximated with acceptable error. The irregular double peak still remains in the central range, which has been published in ref. [22] too.

The problem mentioned above is related to the accuracy of shear viscosity determination, which can be illustrated by the $\ln \eta_{0}-\bar{M}_{m}$ relation. The sensitivity of $\eta_{0}$ to the molecular mass at infinite shear rate is unity $(\alpha=1.0)$, while at zero shear viscosity it is much higher $(\alpha=3.4)$. At $190^{\circ} \mathrm{C}$, depending on the polymer the lowest shear rate, from where extrapolation to zero shear viscosity is performed is $0,5-20 \mathrm{~s}^{-1}$ therefore the empirically found values of $2<\alpha \leq 3.4$ seem to be logical.

Figures 5-7 show the calculated and experimental molecular mass distribution curves (the latter are determined by GPC). The shape of the calculated curves deviates considerably from the experimental ones, and the mentioned anomaly appears on all of them. Serious discrepancies are found in the relative frequencies. In the case of the PVC-4 sample, however, a very good agreement was found.

$\bar{M}_{m}, \bar{M}_{n}$ and $\bar{M}_{m} / \bar{M}_{n}$ values calculated from rheological tests and from the GPC are summarized in Table 6. It has been concluded that the model approximates well the original molecular mass distribution. Unfortunately the same cannot be said of mass and number average molecular masses. Both values are underestimated in most cases, the calcu-

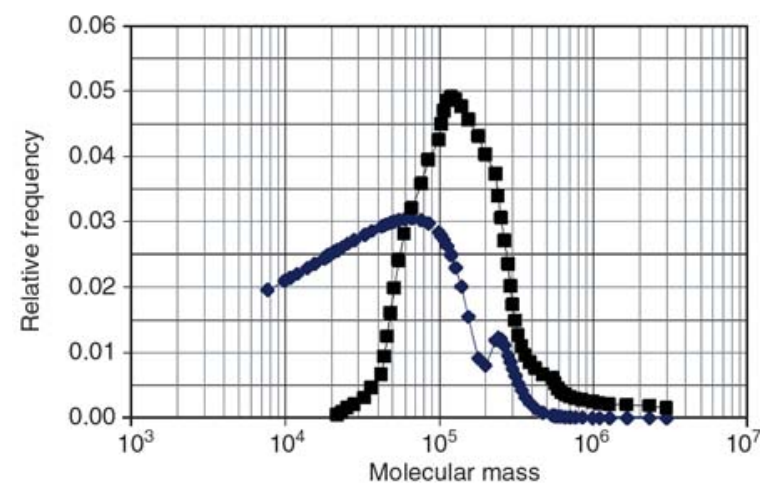

Figure 5. Comparison of calculated $(\diamond)$ and measured (GPC, $\mathbf{a})$ molecular mass distribution curves of sample PS-4

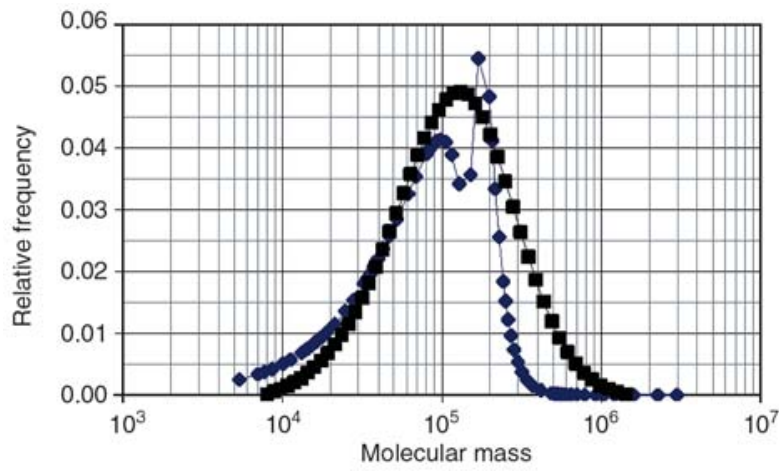

Figure 6. Comparison of calculated $(\downarrow)$ and measured $(\mathrm{GPC}, \mathbf{\square})$ molecular mass distribution curves of sample PVC-4 
Table 6. Comparison of measured and calculated molecular mass distributions of polystyrene, PVC and polypropylene

\begin{tabular}{|c|c|c|c|c|c|c|c|c|c|c|}
\hline \multirow{2}{*}{$\begin{array}{c}\text { Polymer } \\
\text { type }\end{array}$} & \multicolumn{4}{|c|}{ Calculated from rheological tests $(k=1)$} & \multicolumn{3}{|c|}{ Obtained form GPC tests } & \multicolumn{3}{|c|}{ Relative difference* $[\%]^{*}$} \\
\hline & $\alpha$ & $\mathbf{M}_{\mathbf{m}}$ & $\mathbf{M}_{\mathbf{n}}$ & $\bar{M}_{m} / \bar{M}_{n}$ & $\mathbf{M}_{\mathbf{m}}$ & $\bar{M}_{\mathbf{n}}$ & $\bar{M}_{m} / \mathbf{M}_{n}$ & $\overline{\mathbf{M}}_{\mathbf{m}}$ & $\bar{M}_{\mathbf{n}}$ & $\bar{M}_{\mathbf{m}} / \bar{M}_{\mathbf{n}}$ \\
\hline \multicolumn{11}{|c|}{ EDISTIR polystyrene } \\
\hline PS-1 & 2.50 & 73977 & 26623 & 2.779 & 162634 & 64072 & 2.538 & -54.51 & -58.45 & 9.48 \\
\hline PS-2 & 2.60 & 75399 & 29189 & 2.583 & 157532 & 58431 & 2.696 & -52.14 & -50.05 & -4.19 \\
\hline PS-3 & 2.65 & 74803 & 29948 & 2.498 & 160375 & 62151 & 2.580 & -53.36 & -51.81 & -3.19 \\
\hline PS-4 & 2.70 & 79243 & 32754 & 2.419 & 176472 & 71525 & 2.467 & -55.10 & -54.21 & -1.93 \\
\hline PS-5 & 2.80 & 78929 & 34608 & 2.281 & 189487 & 88165 & 2.149 & -58.35 & 60.75 & 6.13 \\
\hline PS-6 & 2.50 & 64685 & 23279 & 2.779 & 158968 & 58993 & 2.695 & -59.31 & -60.54 & 3.11 \\
\hline PS-7 & 2.85 & 83002 & 37403 & 2.219 & 131583 & 59117 & 2.226 & -36.92 & -36.73 & -0.31 \\
\hline PS-8 & 2.50 & 77018 & 27718 & 2.779 & 150708 & 55342 & 2.723 & -48.90 & -49.92 & 2.04 \\
\hline PS-9 & 2.45 & 63067 & 21815 & 2.891 & 187972 & 65458 & 2.873 & -66.45 & -66.67 & 0.63 \\
\hline PS-10 & 2.55 & 71360 & 26662 & 2.676 & 150680 & 55797 & 2.701 & -52.64 & -52.22 & -0.91 \\
\hline PS-11 & 2.50 & 57951 & 20856 & 2.779 & 150798 & 53184 & 2.835 & -61.57 & -60.79 & -1.99 \\
\hline \multicolumn{11}{|c|}{ ONGROLIT PVC } \\
\hline PVC-1 & 3.40 & 53566 & 28208 & 1.899 & 80035 & 40049 & 1.998 & -33.07 & -29.57 & -4.96 \\
\hline PVC-2 & 2.80 & 77283 & 36622 & 2.110 & 106986 & 52384 & 2.042 & -27.76 & -30.09 & 3.34 \\
\hline PVC-3 & 2.55 & 91487 & 43707 & 2.093 & 145541 & 68544 & 2.123 & -37.14 & -36.24 & -1.40 \\
\hline PVC-4 & 2.55 & 111655 & 53689 & 2.080 & 164508 & 80261 & 2.050 & -32.13 & -33.11 & 1.45 \\
\hline PVC-5 & 2.40 & 116814 & 53497 & 2.184 & 188065 & 87418 & 2.151 & -37.89 & -38.80 & 1.51 \\
\hline PVC-6 & 2.30 & 209115 & 100680 & 2.077 & 253923 & 114367 & 2.220 & -17.65 & -11.97 & -6.44 \\
\hline \multicolumn{11}{|c|}{ TIPPLEN polypropylene } \\
\hline PP-1 & 2.050 & 155261 & 34187 & 4.542 & 121850 & 45274 & 4.701 & 27.42 & -24.49 & -3.39 \\
\hline PP-2 & 2.025 & 166486 & 36659 & 4.541 & 219166 & 46200 & 4.744 & -24.04 & -20.65 & -4.27 \\
\hline PP-3 & 1.975 & 116050 & 23800 & 4.876 & 269912 & 55980 & 4.822 & -57.00 & -57.48 & 1.12 \\
\hline PP-4 & 1.950 & 105580 & 20858 & 5.062 & 336073 & 68097 & 4.935 & -68.58 & -69.37 & 2.57 \\
\hline PP-5 & 1.900 & 95560 & 18162 & 5.262 & 480786 & 89048 & 5.399 & -80.12 & -79.60 & -2.55 \\
\hline PP-6 & 1.875 & 122628 & 21480 & 5.709 & 617606 & 107993 & 5.719 & -80.14 & -80.11 & -0.18 \\
\hline
\end{tabular}

*relative difference $=100 \cdot($ calculated from rheological tests - obtained from GPC tests )/(obtained from GPC tests)

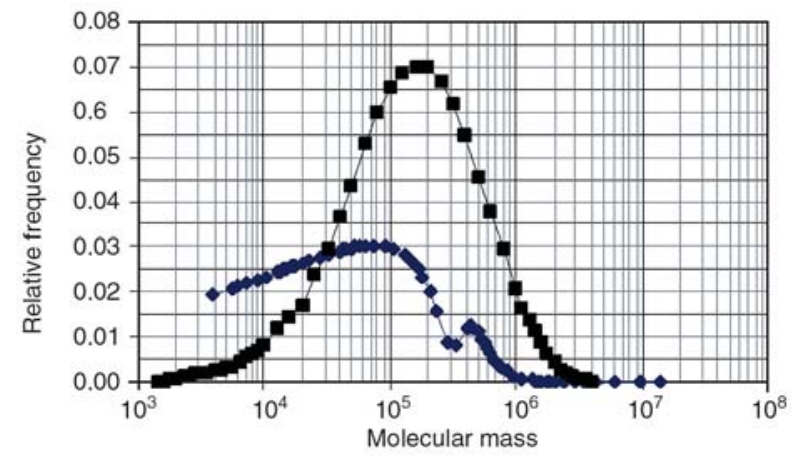

Figure 7. Comparison of calculated $(\diamond)$ and measured (GPC, $\boldsymbol{\square})$ molecular mass distribution curves of sample PP-3 lated values are about half - two third of the values obtained form the GPC tests.

\subsection{Critical evaluation of the applicability of the model}

Table 7. shows how the mass- and number average molecular masses and the distribution itself change with the maximum shear viscosity and with the relaxation time. The data reflect a positive feature of the model, namely

- number average molecular mass $\left(\bar{M}_{n}\right)$ increases parallel with the maximum shear viscosity, i.e.

Table 7. Mass- and number average molecular masses and polydispersities obtained form them

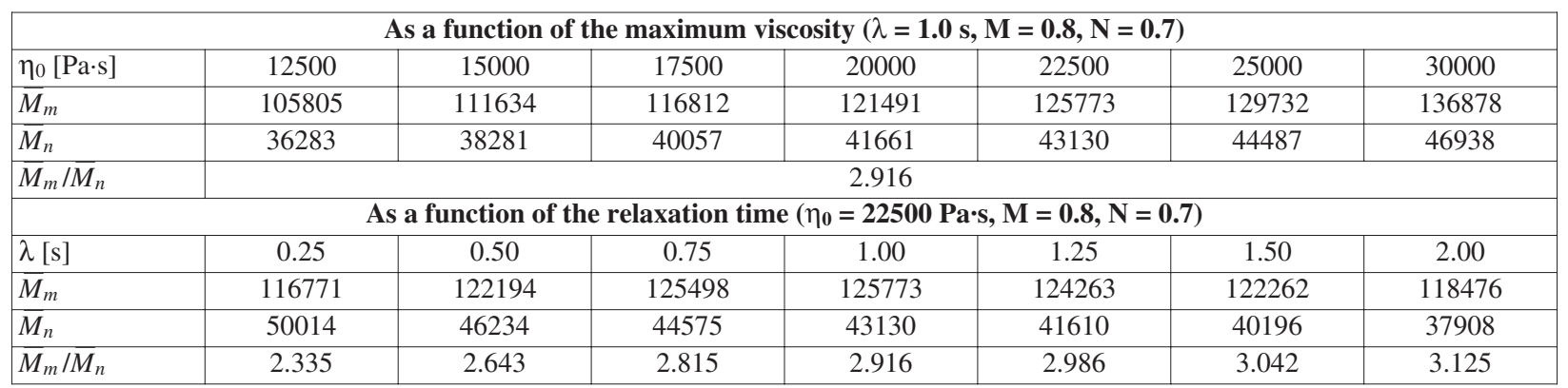


with the mass average molecular mass $\left(\bar{M}_{n}\right)$, the distribution remains constant, if the relaxation time $(\lambda)$ and the Carreau parameters $(M$ and $N)$ remain constant;

- the increase of the relaxation time $(\lambda)$ results in the broadening of the distribution curve if the maximum shear viscosity $\left(\eta_{0}\right)$, and the Carreau parameters $(M$ and $N)$ are kept constant.

A negative feature of the model is that by increasing the $M$ parameter of the Carreau equation results in a gradually narrower distribution, i.e. the algorithm handles the breadth of the molecular mass distribution inversely.

\section{Prediction of the molecular mass distribution of thermoplastics using a model based on the GEX function}

\subsection{Theory}

The method described in Chapter 5. was based on the double derivation of the experimentally determined shear viscosity function. The integral molecular mass distribution function was obtained by processing the first and second derivatives using the described algorithm. Irregularities of the upper branch of the curve and the fact that by increasing the $M$ parameter of the Carreau equation resulted in narrower distribution, i.e. that the algorithm handled the breadth of the molecular mass distribution inversely, prompted us to try the General Exponential (GEX) function, as suggested by Nobile et al. [9], to describe the molecular mass distribution function. It is to be noted, however, that only monomodal distributions with a single peak can be described by the GEX function, shown in Equation (26):

$$
f_{G E X}\left(M ; a, b, M_{0}\right)=\frac{b}{M_{0} \Gamma\left(\frac{a+b}{b}\right)} \cdot\left(\frac{M}{M_{0}}\right)^{a} \cdot e^{-\left(\frac{M}{M_{0}}\right)^{b}}
$$

where $\Gamma(x)$ is the gamma function, $a$ and $b$ are constants, $M$ is the mass average molecular mass, $M_{0}$ is the reference molecular mass.

The starting point is again the experimentally determined shear viscosity function and the four parameters of the Carreau equation $\left(\eta_{0}, \lambda, M\right.$ and $\left.N\right)$ fitted to the experimental points.
The rheological model is defined by a 5-parameter $\left(\eta_{0, G E X}, K_{3 G E X}, a_{G E X}, b_{G E X}\right.$ and $\left.\alpha_{G E X}\right)$ GEX function shown in Equation (28), derived from the assumed form of the molecular mass distribution function (see Equation (27)):

$$
\begin{aligned}
& \left(\frac{\eta}{\eta_{0, G E X}}\right)^{1 / \alpha}= \\
& 1+\frac{\frac{K_{3}}{\dot{\gamma}^{1 / \alpha}} \cdot \Gamma\left(\frac{a+1}{b},\left[\frac{K_{3}}{\dot{\gamma}^{1 / \alpha}}\right]^{b}\right)-\Gamma\left(\frac{a+2}{b},\left[\frac{K_{3}}{\dot{\gamma}^{1 / \alpha}}\right]^{b}\right)}{\Gamma\left(\frac{a+2}{b}\right)}
\end{aligned}
$$

where $\Gamma(x, y)$ in the incomplete gamma function given by Equation (28):

$$
\Gamma(x, y)=\Gamma(x)-\int_{0}^{y} t^{x-1} \cdot e^{-t} \mathrm{~d} t
$$

and $a, b>0$. Notations introduced by Nobile et al. [9] are used in the equations.

\subsection{Calculation of the molecular mass distribution}

The parameters are calculated by fitting Equation (27) to the experimental data using nonlinear regression method.

$M_{1}$ and $M_{0}$ molecular masses necessary for the further calculation are obtained knowing two further parameters $-K_{1}$ and $K_{2}-$ defined by Equations (29) and (30):

$$
\begin{aligned}
& M_{1}=\left(\frac{\eta_{0}}{K_{1}}\right)^{1 / \alpha} \\
& M_{0}=\frac{1}{K_{3} K_{2}^{1 / \alpha}}
\end{aligned}
$$

Molecular mass data of the distribution function are obtained according to Equation (22) where $\eta_{0}$ is the maximum shear viscosity, $K$ is a polymer-specific constant, $\lambda$ is the relaxation time, $\dot{\gamma}$ is the actual shear rate and $k=1$. The corresponding $w(m)_{G E X}$ values are obtained form Equation (26). Of course theses should be normalized according to the $\Sigma w_{i} m_{i}=1$ relation 
The algorithm was tested with PS/PVC/PP samples and it turned out that the calculated distribution curves approximate best those obtained by GPC if $a<b$ and $K_{1}=K_{2}=1-2.25$.

\subsection{Results and discussion. Comparison of the calculated and measured molecular mass distribution}

Figures 8-10 show the molecular mass distribution curves obtained by the GPC method and calculated from shear viscosity function for one PS, PVC a PP sample.

Table 8 presents the constants calculated by the GEX-model for the polymers included in this study, $\bar{M}_{m}, \bar{M}_{n}$ and $\bar{M}_{m} / \bar{M}_{n}$ values, which are compared with those obtained form the GPC tests. Both molecular mass and the distribution values exhibit (with some exceptions) less than $30 \%$ deviation, in the majority of the cases the difference is less than

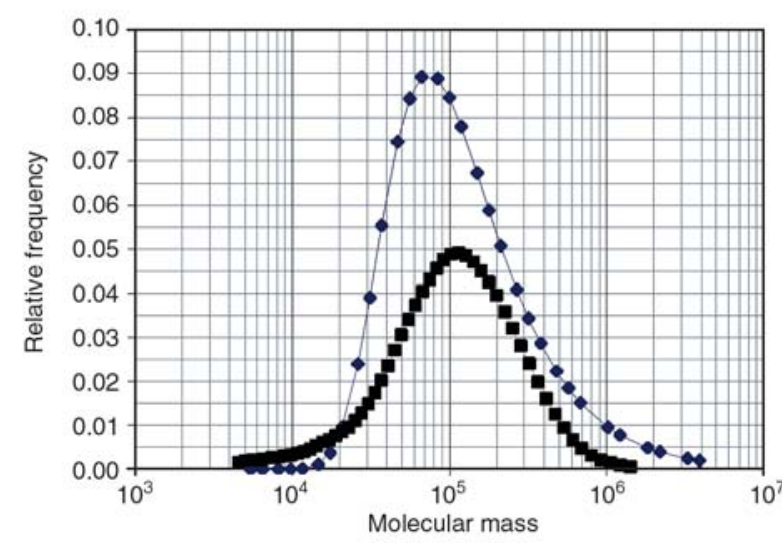

Figure 8. GPC curve of the PS-4 sample (匹) and the molecular mass distribution calculated by the GEX function $(\diamond)$

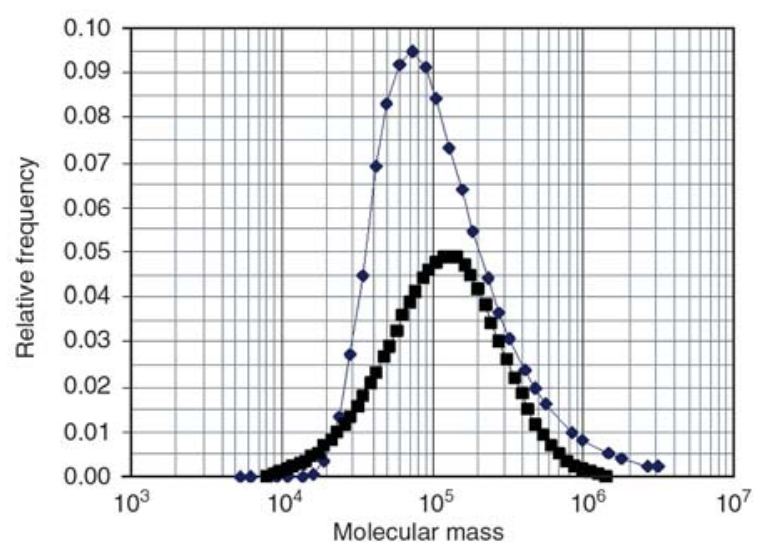

Figure 9. GPC curve of the PVC-4 sample (匹) and the molecular mass distribution calculated by the GEX function $(\diamond)$

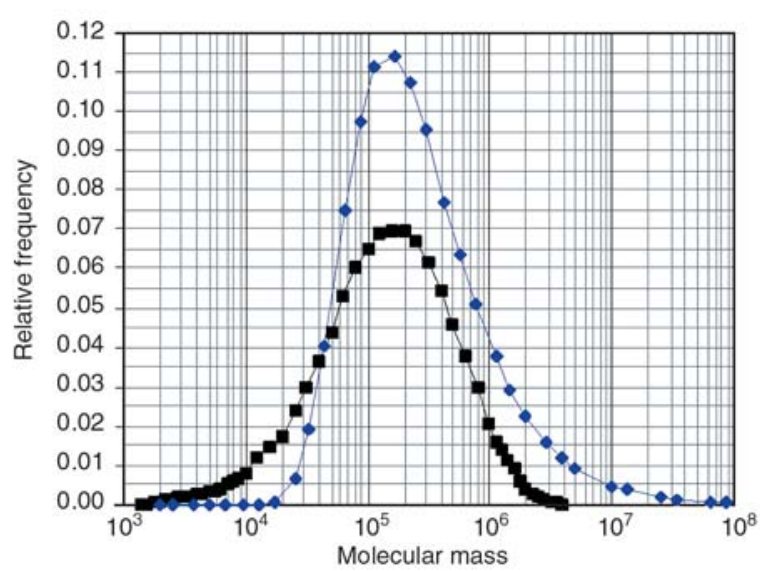

Figure 10. GPC curve of the PP-3 sample (ם) and the molecular mass distribution calculated by the GEX function $(\diamond)$

$10 \%$. Based on these results we can conclude that using the model suggested by us it is possible (within the mentioned limits) it is possible to predict the molecular mass distribution from the rheological data. Individual constants are valid for each polymer, which cannot be predicted on generalized at the present sate of knowledge.

\subsection{Critical evaluation of the applicability of the model}

Taking into account that the breadth of the molecular mass distribution functions of the polymers studied is moderate, it was investigated, whether a systematic variation of the Carreau parameters is reflected in the shape of the GEX distribution function and if yes, how. Therefore four cases were investigated, namely

- $\eta_{0}$ is varied, $\lambda=$ constant, $M=$ constant, and $N=$ constant;

$-\lambda$ is varied, $\eta_{0}=$ constant, $M=$ constant and $N=$ constant;

- $M$ is varied, $\eta_{0}=$ constant, $\lambda=$ constant and $N=$ constant;

$-N$ is varied, $\eta_{0}=$ constant, $\lambda=$ constant and $M=$ constant.

Positive features of the model are as follows:

(1) The increase/decrease of the maximum shear viscosity shifts the curve upwards/downwards along the molecular mass axis, the width of the distribution curve remains constant. 
Table 8. A comparison of the constants of polymers calculated by the GEX model and those obtained from molecular mass distributions calculated from GPC test results

\begin{tabular}{|c|c|c|c|c|c|c|c|c|c|}
\hline \multirow{2}{*}{$\begin{array}{c}\text { Polymer } \\
\text { type }\end{array}$} & \multicolumn{3}{|c|}{ Calculated from the GEX model } & \multicolumn{3}{|c|}{ Calculated from GPC tests } & \multicolumn{3}{|c|}{ Relative difference* [\%] } \\
\hline & $\overline{\mathbf{M}}_{\mathbf{m}}$ & $\overline{\mathbf{M}}_{\mathbf{n}}$ & $\overline{\mathbf{M}}_{\mathbf{m}} / \overline{\mathbf{M}}_{\mathbf{n}}$ & $\overline{\mathbf{M}}_{\mathbf{m}}$ & $\overline{\mathbf{M}}_{\mathbf{n}}$ & $\overline{\mathbf{M}}_{\mathbf{m}} / \overline{\mathbf{M}}_{\mathbf{n}}$ & $\overline{\mathbf{M}}_{\mathbf{m}}$ & $\overline{\mathbf{M}}_{\mathbf{n}}$ & $\overline{\mathbf{M}}_{\mathbf{m}} / \overline{\mathbf{M}}_{\mathbf{n}}$ \\
\hline \multicolumn{10}{|c|}{ EDISTIR polystyrene } \\
\hline PS-1 & 152776 & 60371 & 2.531 & 162634 & 64072 & 2.538 & -6.06 & -5.78 & -0.29 \\
\hline PS-2 & 157475 & 52744 & 2.986 & 157532 & 58431 & 2.696 & -0.04 & -9.73 & 10.76 \\
\hline PS-3 & 171824 & 50492 & 3.404 & 160375 & 62151 & 2.580 & 7.14 & -18.76 & 31.92 \\
\hline PS-4 & 191971 & 77761 & 2.469 & 176472 & 71525 & 2.467 & 8.78 & 8.72 & 0.07 \\
\hline PS-5 & 194411 & 61010 & 3.187 & 189487 & 88165 & 2.149 & 2.60 & -30.80 & 48.29 \\
\hline PS-6 & 294751 & 108468 & 2.717 & 158968 & 58993 & 2.695 & 85.42 & 83.87 & 0.83 \\
\hline PS-7 & 156055 & 77134 & 2.023 & 131583 & 59117 & 2.226 & 18.60 & 30.48 & -9.11 \\
\hline PS-8 & 177085 & 53343 & 3.320 & 150708 & 55342 & 2.723 & 17.50 & -3.61 & 21.91 \\
\hline PS-9 & 216028 & 104523 & 2.067 & 187972 & 65438 & 2.873 & 14.93 & 59.73 & -28.04 \\
\hline PS-10 & 150428 & 72491 & 2.075 & 150680 & 55797 & 2.701 & -0.17 & 29.92 & -23.16 \\
\hline PS-11 & 150765 & 88534 & 1.703 & 150798 & 53184 & 2.835 & -0.02 & 66.47 & -39.94 \\
\hline \multicolumn{10}{|c|}{ ONGROLIT PVC } \\
\hline PVC-1 & 95600 & 48710 & 1.963 & 80035 & 40049 & 1.998 & 19.45 & 21.63 & -1.77 \\
\hline PVC-2 & 92630 & 47868 & 1.935 & 106986 & 52384 & 2.042 & -13.42 & -8.62 & -5.26 \\
\hline PVC-3 & 144287 & 55992 & 2.577 & 145541 & 68544 & 2.123 & -0.86 & -18.31 & 21.37 \\
\hline PVC-4 & 173043 & 78244 & 2.212 & 164508 & 80261 & 2.050 & 5.19 & -2.51 & 7.92 \\
\hline PVC-5 & 191003 & 97504 & 1.959 & 188065 & 87418 & 2.151 & 1.56 & 11.54 & -8.94 \\
\hline PVC-6 & 335544 & 148623 & 2.258 & 253923 & 114367 & 2.220 & 32.14 & 29.95 & 1.70 \\
\hline \multicolumn{10}{|c|}{ TIPPLEN polypropylene } \\
\hline PP-1 & 125357 & 29412 & 4.262 & 121850 & 45274 & 4.701 & 2.88 & -35.04 & -9.34 \\
\hline PP-2 & 236460 & 53324 & 4.434 & 219166 & 46200 & 4.744 & 7.89 & 15.42 & -6.53 \\
\hline PP-3 & 274656 & 63092 & 4.353 & 269912 & 55980 & 4.822 & 1.76 & 12.70 & -9.72 \\
\hline PP-4 & 305930 & 58660 & 5.215 & 336073 & 68097 & 4.935 & -8.97 & -13.86 & 5.67 \\
\hline PP-5 & 526139 & 111638 & 4.713 & 480786 & 89048 & 5.399 & 9.43 & 25.37 & -12.71 \\
\hline PP-6 & 762101 & 122382 & 6.227 & 617606 & 107993 & 5.719 & 23.40 & 13.32 & 8.88 \\
\hline
\end{tabular}

*relative difference $=100 \cdot($ calculated from rheological tests - obtained from GPC tests $) /($ obtained from GPC tests $)$

(2) An increase of the relaxation time results in broader distribution while a decrease leads to a narrower distribution.

(3) Increasing $N$ intensifies the non-Newtonian character and results in a broader distribution, while its decrease causes a more Newtonian character and a narrower distribution function.

Negative characteristics of the model are that

(1) it can predict only narrow and moderately broad $\left(1.5<\bar{M}_{m} / \bar{M}_{n}<6\right)$ distributions,

(2) the variation of $M$ does not cause a significant change in the shape of the distribution function.

\section{Conclusions}

Determining the number- and mass distribution of commercial polystyrene, $\mathrm{PVC}$ and polypropylene samples by GPC method the constants of the distribution functions were calculated. From the shear viscosity function measured at $190^{\circ} \mathrm{C}$ by capillary viscometry the Carreau constants of the polymers were calculated. Equations for the maximum shear viscosity and the mass average molecular mass taken form the literature were compared with our experimental data.

(1) The basic assumption of our model developed to a transform molecular mass distribution to shear viscosity function (direct problem) is that for each shear rate there is a $M_{c}$ critical molecular mass. At $M<M_{c}$ the molecules contribute to the shear viscosity according to the $w_{i} M_{i}$ product, while at $M \geq M_{c}$ according to their mass fraction, as if their molecular mass were $M_{c}$. It is assumed further that the threshold value is independent of the average molecular mass of the sample and of the molecular mass distribution of the whole sample. Measured and calculated shear viscosity functions of the samples mentioned above agree within $10 \%$ error limit.

(2) In order to determine the molecular mass distribution function from the shear viscosity function measured by capillary viscometry (inverse problem) we used the shear viscosity function 
of a two component polymer mixture developed by Bersted. Although the breadth of the distribution functions calculated by the modified Carreau equation approximates well the data derived form the GPC curves, the actual shape of the calculated distribution function and the deviation of measured and calculated mass and number average molecular masses is too high, therefore this model was not developed further.

(3) In order to solve the inverse problem, i.e. for the prediction of the molecular mass distribution a model using the so-called GEX (General Exponential) function is suggested. The rheological model derived from the mathematical form of the distribution function assumed by Nobile is described by a 5-parameter GEX function. Having checked the validity of the model with a large number of polymer samples we concluded that it is possible to predict the molecular mass distribution from the rheological data with an accuracy of $\pm 30 \%$. Individual constants are valid for every polymer, which cannot be generalized at our present state of knowledge.

The positive features of the model include that an increase/decrease of the maximum shear viscosity shifts the curve upwards/downwards the distribution function wile the breadth of the distribution remains constant; the increase of the relaxation time leads to a broader, its increase to a narrower distribution; an increase of $N$ increases the non-Newtonian character and broadens the distribution function, while its decrease decreases the non-Newtonian character and narrows down the distribution function. Negative features of the model include that it can predict only marrow to medium $(1.5<$ $\left.\bar{M}_{m} / \bar{M}_{n}<6\right)$ distributions and a variation of $M$ does not cause a significant change in the shape of the distribution function.

(4) By solving the inverse problem a fast, but scientifically correct method is available for estimating the molecular mass distribution from macroscopic flow data without recourse to a lengthy dissolution process and a GPC test demanding expensive equipment and considerable experience.

\section{Acknowledgements}

The research described here has been performed at the Research Institute for Plastics with the financial support of OTKA T 31898 project 'Relations between polymer structure, molecular mass distribution and viscosity function of thermoplastics'.

\section{References}

[1] Bersted B.: An empirical model relating the molecular weight distribution of high-density polyethylene to the shear dependence of the steady shear melt viscosity. Journal of Applied Polymer Science, 19, 2167-2177 (1975).

DOI: $10.1002 / a p p .1975 .070190810$

[2] Bersted B.: A model relating the elastic properties of high-density polyethylene melts to the molecular weight distribution. Journal of Applied Polymer Science, 20, 2705-2714 (1976). DOI: $\underline{10.1002 / a p p .1976 .070201008}$

[3] Bersted B. H., Slee J. D.: A relationship between steady-state shear melt viscosity and molecular weight distribution in polystyrene. Journal of Applied Polymer Science, 21, 2631-2634 (1977). DOI: $10.1002 / a p p .1977 .070211006$

[4] Malkin A. Ya., Teishev A. E.: Is it possible to determine the molecular mass distribution of a polymer in a unique fashion from the flow curve of its melt? Vysokomolekularnye Soedinenia A, 29, 2230-2234 (1987).

[5] Malkin A. Ya., Teishev A. E.: Method of determination of molecular mass disribution from curves of polymer melt flow. Vysokomolekularnye Soedinenia A, 30, 175-178 (1988)

[6] Malkin A. Ya., Teishev A. E.: Flow curve - molecular weight distribution: Is the solution of the inverse problem possible? Polymer Engineering Science, 31, 1590-1596 (1991). DOI: $10.1002 /$ pen.760312206

[7] Tuminello W. H., Cudré-Maurox N.: Determining molecular weight distributions from viscosity versus shear rate flow curves. Polymer Engineering and Science, 31, 1496-1507 (1991). DOI: $10.1002 /$ pen.760312009

[8] Shaw M. T., Tuminello W. H.: A closer look at the $M W D$-viscosity transform. Polymer Engineering and Science, 34, 159-165 (1994). DOI: $10.1002 /$ pen.760340213

[9] Nobile M. R., Cocchini F., Lawler J. V.: On the stability of molecular weight distributions as computed from the flow curves of polymer melts. Journal of Rheology, 40, 363-382 (1996). DOI: $\underline{10.1122 / 1.550748}$

[10] Provder T., Kuo C. Y.: Recent advances in size exclusion chromatography. Plenum Press, New York (1983). 
[11] Berry G. C., Fox T. G.: The viscosity of polymers and their concentrated solutions. Advances Polymer Science, 5, 261-357 (1968). DOI: $\underline{10.1007 / \mathrm{BFb} 0050985}$

[12] Carreau P. J., De Kee D., Daroux M.: An analysis of the viscous behavior of polymeric solutions. The Canadian Journal of ChemicalEngineering, 57, 135-141 (1979).

[13] Combs R. L., Slonaker D. F., Coover H. W.: Effects of molecular weight distribution and branching on rheological properties of polyolefin melts. Journal of Applied Polymer Science, 13, 519-534 (1969). DOI: $10.1002 / \mathrm{app} .1969 .070130312$

[14] Gahleitner M., Sobczak R.: Bedeutung der Nullviskositätsbestimmung für das Modellieren von Fliesskurven. Kunststoffe, 79, 1213-1216 (1989).

[15] Gahleitner M., Bihlmayer G., Sobczak R.: Modifiziertes Carreau-modell. Kunststoffe, 81, 651-653 (1991).

[16] Mendelson R. A., Bowles W. A., Finger F. L.: Effect of molecular structure on polyethylene melt rheology I: Low-shear behavior. Journal of Polymer Science Part A2: Polymer Physics, 8, 105-126 (1970). DOI: $\underline{10.1002 / \text { pol.1970.160080109 }}$

[17] Zosel A.: Der Einfluss von Molekulargewicht und Molekulargewichtsverteilung auf die viskoelastischen Eigenschaften von Polystyrolschmelzen. Rheologica Acta, 10, 215-224 (1971). DOI: $\underline{10.1007 / \mathrm{BF} 02040445}$
[18] Frank H. P.: Rheologische Messungen an Polypropylenschmelzen. Rheologica Acta, 5, 89-93 (1966).

[19] Shroff R. N., Shida M.: Correlation between steady state flow curve and molecular weight distribution for polyethylene melts. Polymer Engineering and Science, 11, 200-204 (1971). DOI: $10.1002 /$ pen.760110306

[20] Graessley W. W., Segal L.: Effect of molecular weight distribution on the shear dependence of viscosity in polymer systems. AIChE Journal, 16, 261-267 (1970). DOI: $10.1002 /$ aic.690160219

[21] Halász L., Vorster O., Vincze Á.: Relaxation spectrum calculation methods and their use to determine the molecular mass distribution of propylene, ethylene and alfa-olefin copolymers. Progress in Colloid and Polymer Science, 125, 155-160 (2004). DOI: $10.1007 / \mathrm{b} 13435$

[22] Borg T., Pääkkönen E. J.: Linear viscoelastic models: Part II. Recovery of the molecular weight distribution using viscosity data. Journal of Non-Newtonian Fluid Mechanics, 156, 129-138 (2009). DOI: $10.1016 / j . j n n f m .2008 .07 .010$

[23] Friedman E. M., Porter R. S.: Polymer viscositymolecular weight distribution correlation via blending. Transactions of the Society of Rheology, 49, 493 508 (1975). 